\title{
The role of population size in folk tune complexity
}

Sally E. Street ${ }^{1,2,3}$, Tuomas Eerola ${ }^{4}$, Jeremy Kendal ${ }^{1,2,3}$

1. Durham Cultural Evolution Research Centre, Durham University, South Road, Durham, DH1 3LE, U.K.

2. Evolutionary Anthropology Research Group, Department of Anthropology, Durham University, South Road, Durham, DH1 3LE, U.K.

3. Durham Research Methods Centre, Durham University, South Road, Durham, DH1 3LE, U.K.

4. Department of Music, Durham University, Palace Green, Durham, DH1 3RL, U.K.

ORCID identifiers:

Sally Street: https://orcid.org/0000-0001-8939-8016

Tuomas Eerola: https://orcid.org/0000-0002-2896-929X

Jeremy Kendal: https://orcid.org/0000-0001-8644-3117

Author contributions: SES conceived the study, designed the research, collected the data, analysed the data and led on manuscript writing. TE advised and assisted with statistical analysis and contributed to manuscript writing. JK advised on statistical analysis, led the simulation analyses and contributed to manuscript writing. All authors have approved the submitted version and agree to be accountable for their contributions.

Data availability: data and code associated with this study are available from Data Dryad: https://datadryad.org/stash/share/VIle2g1ZCthyuicRaeunaszimKOaR5Yma-SrDtDp6cU

Acknowledgments: we are grateful to Jeremy Keith, founder and curator of The Session website, for helping us with enquiries and access to The Session database.

\begin{abstract}
A positive correlation between population size and cultural complexity is perhaps one of the most consistent findings in the field of cultural evolution. However, previous findings are largely based on studies of technology and are not necessarily generalisable across diverse cultural domains. We investigate the relationship between population size and complexity in music using Irish folk session tunes as a case study. Using analyses of a large online folk tune dataset, we show that tunes played by larger communities of musicians have diversified into a greater number of different versions but are intermediate in melodic complexity. These results suggest that while larger populations create more frequent opportunities for musical innovation, they encourage convergence upon intermediate levels of melodic complexity due to a widespread inverse U-shaped relationship between complexity and aesthetic preference. Our results show that the relationship between population size and cultural complexity is domain-dependent, rather than universal.
\end{abstract}




\section{Introduction}

A positive correlation between population size and cultural complexity is among the most consistent and widely-discussed findings in the field of cultural evolution, supported by multiple lines of evidence (Mesoudi, 2016). Theoretical models suggest that in larger populations, opportunities for innovation are more frequent and beneficial innovations are less likely to be lost due to stochastic effects, supporting diverse cultural repertoires of complex technologies (Henrich, 2004; Kobayashi \& Aoki, 2012; Powell, Shennan, \& Thomas, 2009; Shennan, 2001). These predictions are supported by several historical case studies, such as the substantial reduction in technological repertoire among the indigenous Tasmanian population following their isolation from mainland Australia (Henrich, 2004), and the proliferation of technological innovation along with increases in population density during the Upper Palaeolithic transition in Europe and western Asia (Powell et al., 2009; Shennan, 2001). Comparisons across multiple human populations have also found positive correlations between population size and the diversity and complexity of traditional toolkits (Collard, Ruttle, Buchanan, \& O'Brien, 2013; Kline \& Boyd, 2010). Further, across nonhuman primate species, those with larger social groups have more diverse repertoires of socially learned behaviour, controlling for variation in research effort (Street, Navarrete, Reader, \& Laland, 2017). Finally, transmission chain experiments have shown that larger group sizes help sustain cultural diversity and skill in artificial technological tasks (Derex, Beugin, Godelle, \& Raymond, 2013; Muthukrishna, Shulman, Vasilescu, \& Henrich, 2014).

Support for the positive correlation between population size and cultural complexity is not, however, universal (e.g. (Caldwell \& Millen, 2010; Collard et al., 2005; Vaesen, Collard, Cosgrove, \& Roebroeks, 2016)). Furthermore, previous work has largely focused on case studies from the realm of technology, raising the question of whether the relationship is universal across cultural domains. In the case of folk tales, population size correlates positively with diversity in tale type but negatively with diversity in narrative motifs across Eurasian ethnolinguistic groups (Acerbi, Kendal, \& Tehrani, 2016). These results suggest that larger populations facilitate more frequent opportunities for the invention of new tales, but that more frequent transmission also favours increased compressibility, resulting in a greater number of tales which are on average easier to learn (Acerbi et al., 2016). Similarly, languages with a greater number of speakers have more diverse vocabularies, but simpler grammatical structures (Bromham, Hua, Fitzpatrick, \& Greenhill, 2015; Lupyan \& Dale, 2010; Nettle, 2012; Reali, Chater, \& Christiansen, 2018), and artificial language experiments have shown that larger groups produce communication systems that are simpler and easier for subsequent generations to learn and produce (Fay \& Ellison, 2013). The contrasting findings between studies of language and technology suggest that the relationship between population size and cultural complexity may not be universal, but rather depend on cultural selection pressures specific to different domains (Acerbi et al., 2016; Tamariz, Kirby, \& Carr, 2016).

The relationship between population size and complexity in the domain of music is currently unclear. In common with language and technology, larger populations may facilitate more frequent opportunities for the invention and retention of new musical variants. Alternatively, given that music is largely a communal activity (Savage, Brown, Sakai, \& Currie, 2015; Trehub, Becker, \& Morley, 2015), larger populations may instead reduce diversity by increasing convergence upon fewer 'definitive' versions of melodies. For example, inventing a new version of the "Happy Birthday" song, perhaps the most widely known melody worldwide (Brauneis, 2009), would probably result in correction rather than widespread adoption by others. Larger populations may, further, allow more complex melodies to emerge and spread due to greater variation in player skill level coupled with a tendency to 
copy prestigious, highly skilled players, in line with theoretical models based on technology (Henrich, 2004). Alternatively, in common with language, more frequent transmission in larger populations may result in simplification of melodies towards more learnable forms. The cultural 'fitness landscape' for the arts is, however, distinct from those of both language and technology in that it is determined more strongly by subjective aesthetic preferences, including an enjoyment of innovation and complexity for their own sakes (Tamariz et al., 2016). Aesthetic preferences for complexity, further, appear to trade off against those for predictability and structure, given the frequently observed inverse U-shaped relationship between aesthetic appeal and complexity in the arts, including music (Chmiel \& Schubert, 2017; Delplanque, De Loof, Janssens, \& Verguts, 2019; North \& Hargreaves, 1995; Van Geert \& Wagemans, 2020). Folk music scholars too have suggested that the most popular and enduring session tunes are those with an optimal balance of aesthetic interest and learnability (Hillhouse, 2013; Vallely, 2011), i.e. an intermediate level of complexity.

Folk 'session' tunes provide an ideal case study for investigating the relationship between population size and musical complexity. Session tunes are dance-based melodies of primarily Irish origin, such as jigs, reels and hornpipes, typically performed at informal gatherings (Gagne, 1996; Vallely, 2011). During a session, players take turns to 'lead' sets of tunes, with other players joining in with tunes they recognise (Gagne, 1996; Vallely, 2011). Importantly for the present study, session tunes are almost always performed from memory, meaning that despite the increasing commercialisation and digitalisation of Irish traditional music in recent decades (Gagne, 1996; Hillhouse, 2013; Pendlebury, 2020), individual learning and memory processes still exert considerable influence over session tune innovation and transmission. While there are tens of thousands of folk session tunes in circulation, tunes vary widely in their popularity such that only a relatively small subset enter the 'core' repertoire widely known by most session musicians (Hillhouse, 2013). This means that tunes vary widely in their 'effective cultural population sizes' - i.e. the size of the population of individuals capable of performing a particular cultural trait and potentially transmitting this knowledge to others, analogous to the concept of effective population size in population genetics (Kolodny, Creanza, \& Feldman, 2015; Powell et al., 2009; Shennan, 2001). Tunes also vary widely in their diversity and complexity: while some tunes have diversified into hundreds of different versions ('settings' (Vallely, 2011)), others have only one or two known variants (Roud \& Bishop, 2014). Most session tunes are fairly short, highly structured and relatively easy to learn and remember, following an AABB format comprised of two repeated 8-bar sections (e.g. Morrison's, Drowsy Maggie). However, many are longer and more difficult, including 3-5 or more sections and more technically challenging motifs (e.g. the Frieze Breeches, the Maid at the Spinning Wheen).

Here, we investigate the relationship between population size and complexity using analyses of a large online folk tune dataset, The Session (thesession.org (Keith, 2021)). The Session collection includes a large number $(>38,000)$ of session tune settings available in ABC notation, a convenient format for extraction of measures of melodic complexity measures since no transcription is required. The Session is unique among online folk tune collections in that it records measures of tune popularity. We used two measures of tune popularity as proxies for effective cultural population size: at the level of each tune setting, we used the number of times a setting has been added to a virtual 'set' of tunes typically played together at a session, and at the level of each tune (consisting of one or more settings) we used the number of times a tune has been added to users' virtual 'tunebooks'. We assume that these measures reflect the effective cultural population size for each tune or setting - i.e. the number of players who are able to perform and potentially transmit the tune or setting to other players at real-life sessions. First, to test the alternative predictions that larger effective population sizes either create more frequent opportunities for new tunes to emerge or limit 
157 the frequency of innovation through increased standardisation, we investigate the

158 relationship between tune popularity and a measure of tune diversity, the number of unique

159 settings of each tune documented on the Session website. Secondly, we investigate whether the relationship between population size and musical complexity is positive, negative or inverse U-shaped using several different measures of melodic complexity. Finally, we use a simulation analysis to investigate potential learning mechanisms and population dynamics that may give rise to observed relationships between population size and complexity.

\section{Methods}

\section{Data collection}

We obtained popularity, diversity and complexity data for a large number of traditional session tunes from The Session website (https://github.com/adactio/thesession-data). The Session is a community website dedicated to Irish traditional music, used by session players to identify tunes, find local sessions and contribute to discussion boards. Session website users can create an account which allows them add tunes to their own personal tune library ('tunebook'), add tune settings to virtual 'sets' of tunes intended to be played together at a session and upload new tunes or settings themselves. Irish traditional music is a thriving and evolving genre, comprising both newly composed tunes and tunes that are decades or even centuries old (Hillhouse, 2013). However, users of the Session are explicitly asked to submit traditional tunes rather than their own compositions. Therefore, in theory most of the tunes on the Session website should be of traditional origin, although in practice the origin of session tunes is highly varied and often ambiguous, with new tunes disseminated in multiple ways including both oral tradition and commercial publication (Gagne, 1996; Hillhouse, 2013; Pendlebury, 2020; Vallely, 2011).

We compiled two datasets, one at the level of each individual tune and one at the level of each individual tune setting. We originally obtained setting-level data for all available tunes ( $n=38151$ settings of $n=18042$ tunes, downloaded on 01/02/2021). For tune-level analyses of diversity and popularity, we collapsed this dataset to the tune level, treating diversity as the number of unique settings per tune and popularity as the total number of times a given tune has been added to Session website users' tunebooks. Tune popularity data were available only for tunes added to at least 10 tunebooks ( $n=9782$ tunes). At the setting-level, the measure of popularity available from the Session is the number of times each setting has been added by a website user to a virtual tune set. We excluded settings that had never been added to any sets, leaving setting-level popularity data available for $n=13615$ settings of $n=7089$ tunes (downloaded on 30/04/2021).

\section{Melodic complexity measures}

We estimated the melodic complexity of each tune setting using seven different measures as proxies for the difficulty of learning and performing a tune from memory: number of bars, number of notes, a measure of complexity based on melodic expectations (Eerola, Himberg, Toiviainen, \& Louhivuori, 2006; Eerola \& North, 2000), a measure of complexity based on tone transitions (Simonton, 1984, 1994), first order entropy of pitch class distributions, second order entropy of pitch class distributions (adjacent pitch pairs) and a measure of novelty based on melodic self-similarity (Foote, 2000). The first two measures, number of bars and number of notes, capture tune length, assuming that longer tunes should generally be harder to learn and perform from memory than shorter tunes. The remaining measures capture various dimensions of melodic complexity relevant to the difficulty of learning and correctly recalling melodies. All of the melodic complexity measures have been validated in 
experimental studies, which find that melodies with higher scores are judged as more complex, although measures based on melodic expectations are generally most predictive of listeners' ratings (Eerola et al., 2006; Eerola, Toiviainen, \& Krumhansl, 2002; Eerola \& North, 2000). For all melodic complexity measures, higher values indicate greater complexity and thus lower predictability and greater difficulty to learn.

To count the number of bars in each tune, we first converted tunes from $A B C$ notation, a format commonly used in digital collections of folk tunes (Walshaw, 2011), to MusicXML, which includes bar numbers, using the python script 'abc2xml' (Vree, 2018). We then used string-matching functions in base $R$ (R Core Team, 2020) to extract the last bar number for each tune. Bar counts do not account for repeated sections, therefore a tune consisting of 16 bars with no repeats and a tune comprising two 8-bar repeated sections would both be counted as 16 bars long. This approach is consistent with our study's focus on the difficulty of tunes to learn and play from memory: for example, a tune consisting of two repeated 8-bar sections should not be more difficult to learn and reproduce than a 16-bar tune without repeated sections. Bar counts also do not distinguish between full and partial bars, thus anacruses are counted as a full bar. We excluded a small number of settings $(n=7)$ which were less than 4 bars in length, as they were found to contain tune fragments, corrupted files or incorrectly formatted tunes (e.g. lacking bar divisions).

We obtained all other complexity measures using MIDI toolbox 1.1, a collection of MATLAB functions for extracting melodic variables from MIDI data (Eerola \& Toiviainen, 2016). Before reading tunes into MIDI toolbox, we converted tunes from XML to MIDI format using the Batch Convert plugin (Schmitz, 2020) in MuseScore 3 (Schweer \& MuseScore developer community, 2021). The expectancy-based model (EBM) of melodic complexity attempts to capture the extent to which a melody violates regularities in Western music, taking into account tonal, intervallic and rhythmic factors (Eerola \& North, 2000). For example, small intervals between adjacent notes in a melody are more 'expected' than are large intervals (the principal of proximity), melodic contours are expected to be roughly symmetrical (the principal of registral return) and rhythms are expected to conform to a regular pattern of beats (Eerola \& North, 2000). The tone-transitions model (TTM) attempts to capture melodic originality by measuring the extent to which a melody makes use of rare intervals, based only on tone-transition frequencies (Simonton, 1984, 1994). The two entropy-based measures capture the amount of information in the distribution of pitches and adjacent pairs of pitches (Eerola et al., 2006). Melodic novelty is based on self-similarity (i.e. tunes with low self-similarity are highly novel), calculated by the approach proposed by Foote (Foote, 2000). In this method, the pitch-class content of a windowed segment of a tune is extracted, self-similarity of the pitch-class vectors is computed and the overall novelty is obtained by summing a gaussian checkerboard kernel over the diagonal of the self-similarity matrix (Eerola \& Toiviainen, 2016). Here, we calculate novelty at a resolution of 0.5 beats with a gaussian kernel of 10 beats. Measures could not be extracted for $n=3387$ settings due to corrupt or non-monophonic MIDI files. Four further settings were removed prior to analysis due to formatting issues which caused implausibly high or infinite values. After combining datasets and removing problematic settings, $n=9378$ tunes and $n=12422$ settings remained with complete data on all measures for tune-level and setting-level analyses respectively.

\section{Data analysis}

As our hypotheses concerned the effect of population size on musical diversity and complexity, we treated diversity and complexity measures as dependent variables, predicted by popularity measures. To investigate the effect of tune popularity on diversity, we ran models at the tune level treating diversity (number of unique settings per tune, ' $\mathrm{N}$ settings') 
as the dependent variable and tune popularity (number of adds to virtual tunebooks, ' $\mathrm{N}$ tunebook adds') as a predictor. We also included the number of days since the first version of each tune was uploaded to the Session website as an additional predictor, to control for a potential confound: over time, tunes can only increase in both diversity and popularity. In these models, we checked for collinearity between $\mathrm{N}$ tunebook adds and number of days uploaded by calculating variance inflation factors (VIFs). VIFs were low $(<2)$, suggesting that the independent contribution of both popularity and time uploaded to variation in tune diversity can be robustly estimated. Further, to ensure that this approach adequately controlled for the confounding effect of time since the tunes were uploaded to the site, we conducted simulations to investigate the likelihood of a significant positive association between tune popularity and diversity under a null hypothesis of no direct causal relationship between the two variables, finding no increased risk of type I error (SI Section 1, SI Figures 1-3). Ordinary linear models were not appropriate for analysing the relationship between tune popularity and diversity since the outcome variable, the number of settings per tune, is a count variable with a strongly right-skewed distribution (Crawley, 2014). Exploratory analyses of tune popularity and diversity confirmed that the data violated key assumptions of linear regression models including linearity of relationships and normality and homoscedasticity of residuals (Hector, 2015). We therefore considered two alternative nonlinear models as more appropriate for the data: exponential (a linear model with a log-10 transformed outcome variable) and Poisson models. Both showed qualitatively identical results. We present the results from the exponential model in the main text, and those from the Poisson model in the SI (SI Section 2).

To investigate the effect of tune popularity on complexity, we ran models both at the settinglevel and at the tune-level. In setting-level models, we predicted complexity scores from the setting-level measure of popularity (number of 'set adds'), fitting tune identity as a random effect. In tune-level models, we collapsed complexity scores to the tune level by taking median values across all settings of each tune. Before we examined relationships between population size and melodic complexity, we first explored relationships between the seven different complexity measures to see if it would be appropriate to reduce dimensionality by creating a composite complexity variable. Correlations between the measures were, however, variable and generally low, and a principal components analysis did not suggest a single underlying dimension of melodic complexity (SI Section 3, SI Table 1, SI Figure 4). Therefore, we examined relationships between popularity and all seven measures of melodic complexity in separate analyses. Exploratory plots suggested that larger populations were associated with tunes of intermediate complexity across all measures. Therefore, prior to analysis we transformed all complexity variables to the absolute difference from the median value, such that tunes of intermediate complexity have values of zero and positive values correspond to increases or decreases in complexity relative to the median. If popular tunes are intermediate in complexity, we should expect negative relationships between popularity and deviation from intermediate complexity. Similarly to the analyses of tune popularity and diversity, we found that ordinary linear models were not suitable for analyses of tune popularity and complexity due to highly skewed outcome variables, and used exponential models (with log10-transformed outcome variables) instead. We did not consider using Poisson models for the melodic complexity measures as they are not count variables.

We ran all analyses using the R package MCMCglmm (Hadfield, 2010), using default prior distributions for fixed effects (normal, diffuse with a mean of zero and variance of $10^{8}$ ) and inverse-Wishart priors (setting $V=1$ and $n u=0.002$ ) for random effects (Hadfield, 2021). We ran MCMC chains for a sufficient number of iterations (25000, with a burn-in period of 5000 , sampling every 10 generations) to obtain effective sample sizes of at least 500 for all parameters and confirmed chains had converged on posterior distributions by visually 
examining trace plots and histograms. For all fixed effects, we report the means and 95\% credible intervals from posterior distributions, along with ' $\mathrm{pMCMC}$ ' values (the probability that the posterior mean effect is equal to zero, such that lower pMCMC values indicate stronger evidence for effects in either direction). To measure model fit, we calculate marginal and conditional $\mathrm{R}^{2}$ values, which indicate the proportion of variance explained by the fixed effects only and by both the fixed and random effects respectively (Nakagawa \& Schielzeth, 2013). We also performed cross-validation to assess the models' abilities to predict data outside of the sample. Here, we repeated each model 100 times using a training dataset comprising a random sample of $75 \%$ of the dataset and assessed the model's predictive accuracy for the remaining $25 \%$ of the dataset using root mean squared error (RMSE). We then compared the distribution of RMSE values from the cross-validation procedure with the RMSE for the original model based on the full dataset to summarise predictive performance, reporting the mean and SD for RMSE values from the cross-validation procedure. To simplify interpretation, we normalised RMSE values by dividing them by the range of the dependent variable. Therefore, normalised RMSE values indicate the average error as a proportion of the range of the data from $0-1$, where 0 represents perfect performance and 1 the worst possible performance. We performed cross-validation using custom functions as the MCMCglmm package does not include a built-in function for cross-validation.

\section{Simulation}

We ran a simulation analysis to investigate potential learning processes and population dynamics that may underlie the relationship between population size and complexity in the empirical data. Here, we simulate the diffusion of tune settings in population sizes ranging from 20 to 1,000 in increments of 20 . Each player's initial tune setting has a complexity score drawn from a random normal distribution. Complexity scores of zero represent tunes of intermediate complexity and negative and positive values simpler and more complex tunes respectively. The mean of the starting distribution is shifted by an arbitrary amount (3) away from zero to allow for potential movement towards tunes of intermediate complexity (with $S D=1$ ). At each time-step, there is a small probability $(0.1)$ that each player either randomly selects and copies another player's setting or alters their own setting (with a small amount of error in both cases); otherwise, the player keeps their previous setting. We assume low probabilities of social learning and innovation given that folk music is a fairly conservative tradition in which preservation is emphasised over individual creativity or switching between different tune versions. However, we check that increasing the rates of social learning and innovation does not substantially affect our results (reported in $\mathbf{S I}$ ). We also check that increasing the number of time steps (from 5000 to 50,000) does not affect our main results or conclusions (reported in $\mathbf{S I}$ ).

We compare results across two different social learning conditions, representing random and intermediate-biased copying. Here, we aimed to find out whether a learning bias favouring tunes of intermediate complexity is necessary to explain our results or whether random copying could also potentially give rise to the patterns we observe in the data. Although prior evidence suggests that musicians do have a preference for tunes of intermediate complexity (Chmiel \& Schubert, 2017; Delplanque et al., 2019; North \& Hargreaves, 1995; Van Geert \& Wagemans, 2020), random copying could potentially sustain stable traditions of tunes of intermediate complexity if copied tunes are chosen at random and copying errors that alter tune complexity are random and normally distributed. In both conditions of the simulation, when a player copies another player's setting, they select a tune at random from the population and adopt it with a small amount of copying error drawn from a random normal distribution with a small SD of 0.01. In the random copying condition, the mean of the copying error distribution is zero while in the intermediate-biased condition, the mean is 
0.005 or -0.005 depending on whether the individual's setting is negative or positive, respectively, so that copying error is biased towards intermediate complexity (zero).

We also compared effects across homogeneous and structured populations. Previous theoretical models have shown that population connectivity may be at least as important as size for maintaining complexity as both can increase effective cultural population size (Kolodny et al., 2015; Powell et al., 2009). The community of Session website users is increasingly global and diverse, with players influenced by both local musicians they encounter at real life sessions, and geographically distant musicians that they may only encounter through recordings or, increasingly, online (Gagne, 1996; Hillhouse, 2013). The relative importance of local versus global trends on individual session players' performances and preferences is, however, not yet well investigated. In the homogenous population condition, social influence is global such that players may select any individual in the population to copy. In contrast, in the structured population condition, social influence is local such that individuals select a random individual to copy from a smaller subset of the global population ('local population') of individuals with tune settings that are most similar in complexity to their own. In results reported in the main text, we set local population size to 10 , corresponding to a reasonably realistic number of players at a typical session, but we also check that increasing local population size (to 20) does not qualitatively affect our results (reported in $\mathbf{S I}$ ).

\section{Results}

\section{Tune popularity and diversity}

We find that tune diversity increases with tune popularity, controlling for the effect of time since tunes were uploaded to the Session website (Table 1, Figure 1). The normalised RMSE for the full model predicting diversity from popularity and time since uploaded was 0.186 , meaning that the average prediction error was $\sim 19 \%$ of the range of the data. The mean normalised RMSE from the cross-validation procedure was almost identical $(0.187$, $\mathrm{SD}=0.003$ ) suggesting that the model had good out-of-sample predictive ability. The full model, containing both tune popularity and time since uploaded as predictors, explained twice the amount of variation in tune diversity compared with a reduced model containing time as the only predictor, and $95 \%$ credible intervals for the marginal $R^{2}$ values of each model did not overlap (Table 2).

Exploratory plots showed an apparent, unexpected spike in popularity for tunes uploaded early in the Session website's history (from 2001-2005, SI Figure 1). This pattern may be the result of biases in the curation of the site's collection rather than any effect of user preferences: for example, founders of the site may have prioritised adding highly popular tunes from the 'core' session repertoire at its inception, with more obscure tunes taking longer to be included. However, we also find a positive effect of tune popularity on diversity even when considering only tunes uploaded after the year 2005 (SI Table 2), suggesting that this issue does not cause any spurious associations between popularity and diversity.

\section{Tune popularity and complexity}

In tune-level analyses of popularity and melodic complexity, we find negative effects of tune popularity on deviation from median complexity, i.e. tunes of intermediate complexity are most popular, consistently across all complexity measures (Table 3, Figure 2). The strongest effects are found for measures of complexity based on melodic expectations and 
417 melodic novelty, while number of notes has the weakest effect (Table 3). Marginal $\mathrm{R}^{2}$ values

418 are, however, very low $(<0.1)$, suggesting that many other factors besides popularity determine melodic complexity (Table 3). Normalised RMSE values from the full models were almost identical to mean RMSE values calculated from the cross-validation procedure, suggesting good out-of-sample predictive ability (Table 3). The effects of popularity on complexity at the tune-level were largely limited to tunes uploaded early in the site's history, although the effect of popularity on novelty was similar across the time periods (SI Table 3, 4). Follow-up analyses suggested that these differences are largely driven by the greater popularity of tunes uploaded earlier in the site's history, since we find stronger effects among the most popular than the least popular tunes (SI Table 5, 6). Tunes may therefore need sufficient time (15+ years in the present dataset) to gain enough popularity to result in strong convergence towards intermediate levels of melodic complexity.

In setting-level analyses of popularity and melodic complexity (with tune identity as a random effect), we find similar although less consistent results compared with tune-level analyses. Popular settings have intermediate levels of melodic complexity in terms of bar count, melodic expectations and pitch-pair entropy (Table 4, SI Figure 5). Unlike analyses at the tune-level, effects of popularity on bar count and melodic expectations were fairly consistent across time periods (SI Tables $7, \mathbf{8})$. Marginal $R^{2}$ values were very low $(\leq 0.001)$, while conditional $\mathrm{R}^{2}$ values far higher (0.20-0.70), indicating strong tune-level random effects on setting complexity (Table 4). Normalised RMSE values from the cross-validation procedure were slightly higher than those from the full models, suggesting that the setting-level models had lower out-of-sample predictive ability compared with the tune-level models (Table 4).

Taken together, our empirical results suggest that larger effective cultural population sizes increase the diversity of new melodic variants generated by players, but also result in greater convergence upon tunes of intermediate complexity. Effects are not completely consistent across different levels of analysis, measures of melodic complexity and time periods but this general pattern is evident across our analyses. To confirm this interpretation, we ran a further set of follow-up analyses predicting variation (inter-quartile range) in melodic complexity across all settings of a given tune from popularity and diversity. As expected, we found that variation in melodic complexity increases with the number of settings, but decreases with tune popularity (SI Table 9).

\section{Simulation}

Across all simulation conditions, tune diversity (number of unique settings) increases linearly with population size, due to the increased frequency of innovation in larger populations (Figure 3a). Mean complexity remains constant across population sizes, centred either on the initial population mean (3) if copying is random, or shifted towards 0 if copying is biased towards intermediate complexity (Figure $\mathbf{3 b}$ ). Larger populations have greater variation (SD) in complexity in absolute terms (Figure 3c), which is expected due to sampling effects. However, relative to the number of variants, variation in complexity declines with population size, particularly under the assumption of local social influence (Figure $\mathbf{3 d}$ ). Therefore, in line with our empirical findings, our simulations suggest that larger populations support more innovation and diversification of tunes into more unique variants, but reduce relative variation in complexity, particularly when populations are structured. Random copying can sustain intermediate levels of complexity in tune traditions, but intermediate-biased copying is required for tunes of intermediate complexity to become established in the first place. Figure 4 illustrates typical trends in complexity over time for small $(n=100)$ versus large $(n=1000)$ populations across the four conditions. When social influence is global, variation in complexity declines over time as populations converge towards either the starting mean 
469 (under random copying) or 0 (under biased copying). In contrast, local social influence sustains variation over time, unless overridden by the effect of biased copying. In all four conditions, larger populations sustain more variation in complexity (in absolute terms) over time compared with smaller populations. Under global social influence, smaller populations lose more variation in complexity over time than larger populations, likely due to stochastic effects in smaller populations. However under local social influence, larger populations lose more variation over time since they have more to begin with, particularly when copying is biased towards intermediate complexity. We observe qualitatively the same trends when increasing local population size (SI Figure 6, 7), increasing social learning and innovation rates (SI Figure 8, 9) and increasing the length of simulations (SI Figure 10, 11).

\section{Discussion}

We find that popular folk session tunes are more diverse, but intermediate in melodic complexity. The positive correlation between population size and tune diversity that we identify is consistent with the hypothesis that tunes played by larger communities of musicians have more frequent opportunities to diversify into new versions due to deliberate innovation or copying errors. Therefore, larger effective cultural population sizes support more diverse cultural repertoires in folk music as they do in other domains including technology and language (Acerbi et al., 2016; Bromham et al., 2015; Collard et al., 2013; Henrich, 2004; Kline \& Boyd, 2010; Kobayashi \& Aoki, 2012; Lupyan \& Dale, 2010; Nettle, 2012; Powell et al., 2009; Reali et al., 2018; Shennan, 2001). Together with our simulations which consistently showed that larger populations produce more tune variants as a result of more frequent innovation, these results suggest that the positive relationship between population size and innovation rate generalises across diverse contexts. The inverse- $U$ shaped relationship that we identify between popularity and melodic complexity, however, differs from both the positive and negative relationships previously found for technological and linguistic complexity respectively (Acerbi et al., 2016; Bromham et al., 2015; Collard et al., 2013; Kline \& Boyd, 2010; Lupyan \& Dale, 2010; Nettle, 2012; Reali et al., 2018). Therefore, our findings confirm that the relationship between population size and cultural complexity is not universal, but rather depends on selective pressures specific to different cultural domains (Acerbi et al., 2016; Tamariz et al., 2016).

Our findings suggest that a detailed understanding of how cultural fitness landscapes vary between different domains, particularly in terms of the relative benefits of innovation, is essential for understanding the cultural evolution of complexity. Efficiency generally trumps aesthetic concerns in the domains of language and technology, favouring maximum compressibility, but innovation is less constrained in the arts. In support of this explanation, a transmission chain study showed that innovation and increases in complexity were most frequent when an experimental task was framed as an aesthetic rather than technological or linguistic challenge (Tamariz et al., 2016). The optimal balance between familiarity and innovation in artistic domains will depend, further, on distinct features of specific artistic genres and social contexts. While improvisation is important in, for example, jazz and North Indian classical music, folk music is by nature relatively conservative, with individual innovation limited by an emphasis on preserving traditional tunes (Vallely, 2011) and by the constraints of typical folk tune structure, phrasing and tonality (Savage, Chiba, Currie, Suzuki, \& Atkinson, 2020). The finding that session tunes of intermediate complexity are most popular is consistent with previous evidence that preferences for novelty in music trade off against preferences for predictability (Chmiel \& Schubert, 2017; Delplanque et al., 2019; North \& Hargreaves, 1995; Van Geert \& Wagemans, 2020), confirming the suggestion of folk scholars that tunes with an optimal balance of playability and aesthetic interest are most 
521 likely to become established favourites (Hillhouse, 2013; Vallely, 2011). While our simulations showed that intermediate levels of tune complexity are maintained by unbiased learning over time, an active preference for intermediate complexity is required for initially

Larger populations, therefore, do not necessarily increase or decrease complexity but rather increase diversity, providing greater potential for selection towards optimal levels of complexity for a given trait. In this way, our empirical results are consistent with classic population genetics models which show that larger populations generate more variation and thus potential for stronger selection towards the optimum phenotype for a given environment, while stochastic processes dominate in smaller populations (Bromham et al., 2015; Collard et al., 2005, 2013; Shennan, 2001).Our simulations, however, suggest that biological evolutionary mechanisms do not necessarily generalise to all cultural evolutionary contexts. When we assume that social influence is global, small populations actually lose more variation over time compared with larger populations (Figure 4), likely because there is less innovation to compensate for the global convergence caused by social learning. However, the reverse is true when we model structured populations in which individuals learn from others who possess cultural variants most similar to their own. Local influence sustains more variation in populations than global influence as it prevents variation collapsing into a small number of dominant 'traditions', and since larger populations have more variation to begin with, they have more to lose, at least when copying is biased towards intermediate levels of complexity. Although the relative importance of local versus global trends on session musicians is not well understood, individuals are probably more strongly influenced by the musicians they encounter most frequently at real-life sessions and therefore the local social influence condition likely best reflects real-life population dynamics among session musicians. Therefore, taken together, the empirical and simulation results are consistent with more recent genetic models suggesting that the strength of selection does not always increase with effective population size, but rather depends on various contextual factors including underlying mutation processes and population dynamics (Lanfear, Kokko, \& Eyre-Walker, 2014). Our simulation results are not necessarily specific to folk music but can potentially apply to any continuously distributed trait subject to social learning processes similar to those specified in the model.

Evolutionary approaches have a long and controversial history in the study of folk music. While early folk song researchers, such as Cecil Sharp and Béla Bartók, viewed folk song traditions in explicitly evolutionary terms (Bennett, 2016; Pendlebury, 2020), ethnomusicologists have now largely abandoned evolutionary approaches in response to abuses of evolutionary theory to justify racist and colonialist assumptions about the cultural superiority of Western music (Savage, 2019). However, researchers in the modern field of cultural evolution completely reject Spencerian, progressivist models of evolution which have no basis in biological reality (Mesoudi, 2011), and a renewed interest in cultural evolutionary approaches to music has recently begun to emerge (e.g. (Mehr et al., 2019; Ravignani, Delgado, \& Kirby, 2016; Savage, 2019). Even outside the field of cultural evolution, Irish traditional music is often discussed in implicitly evolutionary terms. For example, the Companion to Irish Traditional Music (Vallely, 2011) describes how new tunes enter the core repertoire as follows: "Communities of traditional musicians tend to vote collectively with their fingers. In a largely unspoken process of selection, a minority of tunes possessed of that special combination of playability and aesthetic interest gradually unfold themselves into the traditional repertoire[...]". Our findings fit exactly with this characterisation and show that a preference for melodies of intermediate complexity previously demonstrated in laboratorybased experimental studies (Chmiel \& Schubert, 2017; Delplanque et al., 2019; North \& Hargreaves, 1995; Van Geert \& Wagemans, 2020) affects large-scale naturally-occurring 
573 trends in folk music. We therefore demonstrate that a cultural evolutionary framework can

574 make a useful contribution to the study of folk music by linking population-level trends with

575 individual psychological factors, and hope that our findings will generate more interest in

576 cultural evolutionary approaches among folk scholars. Further investigations of the role of

577 population size in complexity across multiple, cross-cultural folk music corpora would be of

578 great value in understanding the extent to which psychological factors shaping trends in folk

579

580

581

582

583

584

585

586

587

588

589

590

591

592

593

594

595

596

597

598

599

600

601

602

603

604

605

606

607

608

609

610

611

612

613

614

615

616

617

618

619

620

621

622

623

Although we identify patterns consistent with causal effects of population size on complexity in music in our empirical data, we must acknowledge that our analyses are correlational in nature and therefore that alternative explanations are possible. In particular, popularity may be influenced by melodic complexity rather than the other way around. Given the commonly inverse-U shaped relationship between melodic complexity and musical appeal (Chmiel \& Schubert, 2017; Delplanque et al., 2019; North \& Hargreaves, 1995; Van Geert \& Wagemans, 2020), users of the Session website likely prefer to add tunes of intermediate complexity to their tunebooks. Therefore, our results are consistent both with a causal effect of effective cultural population size on folk tune complexity and of folk tune complexity on effective cultural population size. In fact, changes in complexity and effective population size could reinforce one another in a runaway process, a possibility that has not yet been investigated in theoretical analyses (Kolodny et al., 2015). Similarly, while we focus on the possibility that that larger communities of players create more frequent opportunities for tunes to diversify, the reverse causal scenario is possible due to sampling biases (Acerbi et al., 2016) - popular tunes may have more versions recorded on the Session than unpopular tunes because more effort is made to catalogue their diversity. Finally, there is some uncertainty about the extent to which the tune popularity measures we obtain from the Session are good proxies for the effective cultural population size of each tune. Presumably, when a user adds a tune to their virtual tune book or a setting to a virtual set, they like the tune and have some interest in performing it, but it does not necessarily mean that they will actually learn and potentially transmit it to others. Our simulations, however, show that our empirical results are consistent with what we would expect to see if our popularity measures do in fact reflect effective population sizes and influence trends in tune complexity over time. We hope that our findings will inspire future experimental studies on the role of population size in musical complexity, allowing for more in-depth investigation of causal processes.

We have shown that popular folk tunes have a greater number of different versions and tend to be intermediate in melodic complexity. These results suggest that tunes played by larger communities of musicians, i.e. those with larger effective cultural population sizes, have more frequent opportunities to diversify and converge more strongly towards an optimum balance of playability and aesthetic interest. The relationship between population size and cultural complexity for music is distinct from that found previously for both language and technology, most likely due to the relative lack of functional constraints on innovation within the arts. While the positive correlation between population size and cultural complexity is one of the most consistent findings in cultural evolutionary research (Mesoudi, 2016), the present results add to suggestions that fundamental cultural evolutionary processes in fact operate very differently across different domains (Nettle, 2017; Sperber, 1996; Tamariz et al., 2016). Therefore, an understanding of how cultural evolutionary dynamics vary across domains is crucial for a more generally applicable theory of cultural evolution and researchers should not assume that processes identified in studies of technology or language will necessarily generalise to other domains, particularly the arts. This study, together with a welcome recent proliferation of cultural evolutionary studies in music (e.g.(Mehr et al., 2019; Ravignani et al., 2016; Savage, 2019)), highlights the importance of 
considering the full spectrum of human cultural activities for a comprehensive understanding of cultural evolution.

\section{References}

Acerbi, A., Kendal, J., \& Tehrani, J. J. (2016). Cultural complexity and demography: The case of folktales. Evolution and Human Behavior, 38(4), 474-480. https://doi.org/10.1016/j.evolhumbehav.2017.03.005

Bennett, J. (2016). Béla bartók's evolutionary model of folk music. Twentieth-Century Music, 13(2), 291-320. https://doi.org/10.1017/S1478572216000062

Brauneis, R. (2009). Copyright and the world's most popular song. Journal of the Copyright Society of the U.S.A., 56(2-3), 335-426. https://doi.org/10.2139/ssrn.1111624

Bromham, L., Hua, X., Fitzpatrick, T. G., \& Greenhill, S. J. (2015). Rate of language evolution is affected by population size. Proceedings of the National Academy of Sciences of the United States of America, 112(7), 2097-2102. https://doi.org/10.1073/pnas.1419704112

Caldwell, C. a, \& Millen, A. E. (2010). Human cumulative culture in the laboratory: Effects of (micro) population size. Learning \& Behavior, 38(3), 310-318. https://doi.org/10.3758/lb.38.3.310

Chmiel, A., \& Schubert, E. (2017). Back to the inverted-U for music preference: A review of the literature. Psychology of Music, 45(6), 886-909. https://doi.org/10.1177/0305735617697507

Collard, M., Kemery, M., Banks, S., Canadian, S., Journal, A., Collard, M., ... Banks, S. (2005). Causes of Toolkit Variation Among Hunter-Gatherers: A Test of Four Competing Hypotheses Published by: Canadian Archaeological Association Stable URL : http://www.jstor.org/stable/41103514 REFERENCES Linked references are available on JSTOR for this arti. Canadian Journal of Archaeology, 29(1), 1-19.

Collard, M., Ruttle, A., Buchanan, B., \& O’Brien, M. J. (2013). Population Size and Cultural Evolution in Nonindustrial Food-Producing Societies. PLOS ONE, 8(9), 1-6. https://doi.org/10.1371/journal.pone.0072628

Crawley, M. J. (2014). Statistics: An Introduction Using R (2nd ed.). John Wiley \& Sons, Inc. Delplanque, J., De Loof, E., Janssens, C., \& Verguts, T. (2019). The sound of beauty: How complexity determines aesthetic preference. Acta Psychologica, 192(March 2018), 146-152. https://doi.org/10.1016/j.actpsy.2018.11.011

Derex, M., Beugin, M.-P., Godelle, B., \& Raymond, M. (2013). Experimental evidence for the influence of group size on cultural complexity. Nature, 503(7476), 389-391. https://doi.org/10.1038/nature12774

Eerola, T., Himberg, T., Toiviainen, P., \& Louhivuori, J. (2006). Perceived complexity of western and African folk melodies by western and African listeners. Psychology of Music, 34(3), 337-371. https://doi.org/10.1177/0305735606064842

Eerola, T., \& North, A. C. (2000). Proceedings paper Expectancy-Based Model of Melodic Complexity, 1-7. Retrieved from http://citeseerx.ist.psu.edu/viewdoc/summary?doi=10.1.1.110.3186

Eerola, T., \& Toiviainen, P. (2016). MIDI Toolbox 1.1. Retrieved from https://www.jyu.fi/hum/laitokset/musiikki/en/research/coe/materials/miditoolbox/Manual \%5Cnpapers3://publication/uuid/51F2F111-EF19-4737-B84B-B5F8565A471B

Eerola, T., Toiviainen, P., \& Krumhansl, C. L. (2002). Real-Time Prediction of Melodies: Continuous Predictability Judgements and Dynamic Models. Proceedings of the 7th International Conference on Music Perception and Cognition, 0, 473-476. Retrieved from http://www.cc.jyu.fi/ ptee/publications/2_2002.pdf

Fay, N., \& Ellison, T. M. (2013). The Cultural Evolution of Human Communication Systems in Different Sized Populations: Usability Trumps Learnability. PLoS ONE, 8(8). 
https://doi.org/10.1371/journal.pone.0071781

Foote, J. (2000). Automatic audio segmentation using a measure of audio novelty. In Proceedings of IEEE international conference on multimedia and expo (ICME) (pp. 452-455).

Gagne, R. (1996). From Session to CD: Recent Influences on Irish Traditional Dance Music in America.

Hadfield, J. D. (2010). MCMC methods for multi-response generalized linear mixed models: The MCMCglmm R package. Journal of Statistical Software, 33(2), 1-22.

Hadfield, J. D. (2021). MCMCglmm course notes. Retrieved from https://cran.rproject.org/web/packages/MCMCglmm/vignettes/CourseNotes.pdf

Hector, A. (2015). The New Statistics in R: An Introduction. Oxford: Oxford University Press.

Henrich, J. (2004). Demography and cultural evolution: how adaptive cultural processes can produce maladaptive losses - the Tasmanian case. American Antiquity, 69(2), 197-214.

Hillhouse, A. (2013). Hooks and New Tunes: Contemporary Irish Dance Music in Its Transnational Context, 3(July), 38-60.

Jacoby, N., \& McDermott, J. H. (2017). Integer Ratio Priors on Musical Rhythm Revealed Cross-culturally by Iterated Reproduction. Current Biology, 27(3), 359-370. https://doi.org/10.1016/j.cub.2016.12.031

Keith, J. (2021). The Session. Retrieved January 21, 2021, from https://thesession.org Kline, M. A., \& Boyd, R. (2010). Population size predicts technological complexity in Oceania. Proceedings. Biological Sciences / The Royal Society, 277(1693), 25592564. https://doi.org/10.1098/rspb.2010.0452

Kobayashi, Y., \& Aoki, K. (2012). Innovativeness, population size and cumulative cultural evolution. Theoretical Population Biology, 82(1), 38-47. https://doi.org/10.1016/j.tpb.2012.04.001

Kolodny, O., Creanza, N., \& Feldman, M. W. (2015). Evolution in leaps: The punctuated accumulation and loss of cultural innovations. Proceedings of the National Academy of Sciences of the United States of America, 112(49), E6762-E6769. https://doi.org/10.1073/pnas.1520492112

Lanfear, R., Kokko, H., \& Eyre-Walker, A. (2014). Population size and the rate of evolution. Trends in Ecology and Evolution, 29(1), 33-41. https://doi.org/10.1016/j.tree.2013.09.009

Lupyan, G., \& Dale, R. (2010). Language structure is partly determined by social structure. PLOS ONE, 5(1). https://doi.org/10.1371/journal.pone.0008559

Mehr, S. A., Singh, M., Knox, D., Ketter, D. M., Pickens-Jones, D., Atwood, S., ... Glowacki, L. (2019). Universality and diversity in human song. Science (New York, N.Y.), 366(6468). https://doi.org/10.1126/science.aax0868

Mesoudi, A. (2011). Cultural Evolution. Chicago: Chicago University Press.

Mesoudi, A. (2016). Cultural Evolution: A Review of Theory, Findings and Controversies. Evolutionary Biology, 43(4), 481-497. https://doi.org/10.1007/s11692-015-9320-0

Muthukrishna, M., Shulman, B. W., Vasilescu, V., \& Henrich, J. (2014). Sociality influences cultural complexity, (November 2013).

Nakagawa, S., \& Schielzeth, H. (2013). A general and simple method for obtaining R 2 from generalized linear mixed-effects models. Methods in Ecology and Evolution, 4(2), 133142. https://doi.org/10.1111/j.2041-210x.2012.00261.x

Nettle, D. (2012). Social scale and structural complexity in human languages. Philosophical Transactions of the Royal Society B: Biological Sciences, 367(1597), 1829-1836. https://doi.org/10.1098/rstb.2011.0216

Nettle, D. (2017). What is cultural evolution like? Retrieved from https://www.danielnettle.org.uk/2017/10/30/hotte-8-cultural-evolution/

North, A. C., \& Hargreaves, D. J. (1995). Subjective complexity, familiarity, and liking for popular music. Psychomusicology: A Journal of Research in Music Cognition, 14(1-2), 
Pendlebury, C. (2020). Tune Families and Tune Histories. Folk Music Journal, 11(5), 9684.

Powell, A., Shennan, S., \& Thomas, M. G. (2009). Late Pleistocene demography and the appearance of modern human behavior. Science (New York, N.Y.), 324(5932), 12981301. https://doi.org/10.1126/science. 1170165

R Core Team. (2020). R: A language and environment for statistical computing. Version 3.6.3. Vienna, Austria: R Foundation for Statistical Computing. Retrieved from http://www.r-project.org/

Ravignani, A., Delgado, T., \& Kirby, S. (2016). Musical evolution in the lab exhibits rhythmic universals. Nature Publishing Group, 0007(December), 1-7. https://doi.org/10.1038/s41562-016-0007

Reali, F., Chater, N., \& Christiansen, M. H. (2018). Simpler grammar, larger vocabulary: How population size affects language. Proceedings of the Royal Society B: Biological Sciences, 285(1871). https://doi.org/10.1098/rspb.2017.2586

Roud, S., \& Bishop, J. (2014). The New Penguin Book of English Folk Songs. (Steve Roud \& J. Bishop, Eds.). London: Penguin Classics.

Savage, P. E. (2019). Cultural evolution of music. Palgrave Communications, 5(1), 16. https://doi.org/10.1057/s41599-019-0221-1

Savage, P. E., Brown, S., Sakai, E., \& Currie, T. E. (2015). Statistical universals reveal the structures and functions of human music. Proceedings of the National Academy of Sciences, 112(29), 8987-8992. https://doi.org/10.1073/pnas.1414495112

Savage, P. E., Chiba, G., Currie, T. E., Suzuki, H., \& Atkinson, Q. (2020). Sequence alignment of folk song melodies reveals cross-cultural mechanisms of musical evolution. PsyArXiv. Retrieved from https://doi.org/10.31234/osf.io/5rj6y

Schmitz, J. (2020). Batch Convert. Retrieved from https://musescore.org/en/project/batchconvert

Schweer, W., \& MuseScore developer community. (2021). MuseScore 3.6.2. Retrieved from https://musescore.org/en

Shennan, S. (2001). Demography and cultural innovation: A model and its implications for the emergence of modern human culture. Cambridge Archaeological Journal, 11(1), 516. https://doi.org/10.1017/s0959774301000014

Simonton, D. K. (1984). Melodic Structure and Note Transition Probabilities: A Content Analysis of 15,618 Classical Themes. Psychology of Music, 12(1), 3-16. https://doi.org/10.1177/0305735684121001

Simonton, D. K. (1994). Computer Content Analysis of Melodic Structure: Classical Composers and Their Compositions. Psychology of Music, 22(1), 31-43. https://doi.org/10.1177/0305735694221003

Sperber, D. (1996). Explaining Culture: A Naturalistic Approach. Oxford: Blackwell Publishing.

Street, S. E., Navarrete, A. F., Reader, S. M., \& Laland, K. N. (2017). Coevolution of cultural intelligence, extended life history, sociality, and brain size in primates. Proceedings of the National Academy of Sciences of the United States of America, 114(30), 79087914. https://doi.org/10.1073/pnas.1620734114

Tamariz, M., Kirby, S., \& Carr, J. W. (2016). Cultural Evolution Across Domains: Language, Technology and Art. Proceedings of the 38th Annual Conference of the Cognitive Science Society, 2765-2770.

Trehub, S. E., Becker, J., \& Morley, I. (2015). Cross-cultural perspectives on music and musicality. Philosophical Transactions of the Royal Society of London. Series B, Biological Sciences, 370(1664), 20140096. https://doi.org/10.1098/rstb.2014.0096 Vaesen, K., Collard, M., Cosgrove, R., \& Roebroeks, W. (2016). Population size does not explain past changes in cultural complexity. Proceedings of the National Academy of Sciences of the United States of America, 113(16), E4241-E4247. 
https://doi.org/10.1073/pnas.1520288113

Vallely, F. (2011). The Companion to Irish Traditional Music. (F. Vallely, Ed.) (2nd ed.). Cork, Ireland: Cork University Press.

Van Geert, E., \& Wagemans, J. (2020). Order, complexity, and aesthetic appreciation. Psychology of Aesthetics, Creativity, and the Arts, 14(2), 135-154. https://doi.org/10.1037/aca0000224

Vree, W. (2018). abc2xml. Retrieved December 10, 2018, from https://wim.vree.org/svgParse/abc2xml.html

Walshaw, C. (2011). The abc music standard 2.1. Retrieved March 17, 2021, from http://abcnotation.com/wiki/abc:standard:v2.1 
836

837

838

839

840

841

842

843

844

845

846

847

848

849

850

851

852

853

854

855

856

857

858

859

860

861

862

863

864

865

866

867

868

869

870

871

872

873

874

Table 1

\begin{tabular}{|l|r|r|r|r|}
\hline Parameter & \multicolumn{1}{|c|}{ Post. means } & \multicolumn{1}{|l}{ I-95\% Cl } & \multicolumn{1}{l|}{ U-95\% Cl } & \multicolumn{1}{l|}{ PMCMC } \\
\hline $\mathrm{N}$ tunebook adds & 0.00052 & 0.00050 & 0.00055 & $<0.001$ \\
\hline Days since uploaded & 0.00005 & 0.00005 & 0.00006 & $<0.001$ \\
\hline
\end{tabular}

Results of regression model predicting diversity ( $N$ settings) from popularity ( $N$ tunebook adds) and days since uploaded to the site ( $n=9378$ tunes, marginal $R^{2}=0.26$, normalised RMSE for full model $=0.186$, mean normalised $R M S E$ from cross-validation $=0.187, S D=0.003)$. Post. means $=$ mean estimates from posterior distributions, $1-95 \% \mathrm{Cl}=$ lower $95 \%$ credible intervals from posterior distributions, $u-95 \% \mathrm{Cl}=$ upper $95 \%$ credible intervals from posterior distributions and $\mathrm{pMCMC}=$ 'pMCMC value'.

\section{Table 2}

\begin{tabular}{|l|l|l|l|}
\hline Model & Post. means & I-95\% Cl & u-95\% Cl \\
\hline Full model (N tunebook adds + days uploaded) & 0.26 & 0.24 & 0.27 \\
\hline Reduced model (days uploaded only) & 0.13 & 0.12 & 0.14 \\
\hline
\end{tabular}

Comparison of marginal $R^{2}$ values for the full model $(n=9378$ ), including both popularity ( $N$ tunebook adds) and days since uploaded as predictors, and the reduced model, including only days since uploaded as a predictor. Post. means = mean estimates from posterior distributions, $1-95 \% \mathrm{Cl}=1$ lower 95\% credible intervals from posterior distributions and u-95\% Cl = upper $95 \%$ credible intervals from posterior distributions.

\section{Table 3}

\begin{tabular}{|c|c|c|c|c|c|c|c|}
\hline Outcome variable & Post. mean & $\mathrm{I}-95 \% \mathrm{Cl}$ & $\mathrm{u}-95 \% \mathrm{Cl}$ & pMCMC & Marg. $\mathbf{R}^{2}$ & RMSE & CV RMSE \\
\hline Bar count & -0.000086 & -0.000121 & -0.000047 & $<0.001$ & 0.002 & 0.210 & $0.210[0.002]$ \\
\hline Note count & & & & 0.070 & $<0.001$ & 0.148 & $0.148[0.002]$ \\
\hline Melodic expectations & -0.000027 & -0.000033 & .000020 & $<0.001$ & 0.008 & 0.152 & $0.152[0.003]$ \\
\hline Tone transitions & -0.000015 & -0.000024 & -0.000003 & 0.007 & 0.001 & 0.176 & $0.176[0.002]$ \\
\hline & & & & & 0.002 & 110 & $0.110[0.002]$ \\
\hline Pitch-pair entropy & -0.0 & -0.000004 & -0.000002 & $<0.001$ & 0.004 & 0.138 & $0.138[0.002]$ \\
\hline Novelty & -0.000005 & -0.000006 & -0.000004 & $<0.001$ & 0.007 & 0.150 & $0.149[0.002]$ \\
\hline
\end{tabular}

Results of tune-level models predicting measures of tune complexity from popularity ( $N$ tunebook adds, $n=9378$ ). Post. means = mean estimates from posterior distributions, $1-95 \% \mathrm{Cl}=1$ lower $95 \%$ credible intervals from posterior distributions, $u-95 \% \mathrm{Cl}=$ upper $95 \%$ credible intervals from posterior distributions, $p M C M C=$ ' $p M C M C$ value', marg. $R^{2}=$ marginal $R^{2}$, the proportion of variance explained by the fixed effects in the models, RMSE = normalised root mean squared error for the full model and CV RMSE = mean and SD from the distribution of normalised root mean squared error values from the CV models. All melodic complexity measures were re-scaled as the absolute deviation from the median prior to analysis, where low values indicate tunes close to median complexity. Therefore, negative coefficients here indicate that as popularity increases, tunes move towards intermediate levels of complexity. 


\begin{tabular}{|l|r|r|r|r|r|r|r|r|}
\hline Outcome variable & \multicolumn{1}{|l|}{ Post. mean } & \multicolumn{1}{l}{ I-95\% Cl } & \multicolumn{1}{l|}{ U-95\% Cl } & \multicolumn{1}{l|}{ pMCMC } & Marg. R & \multicolumn{1}{c|}{ Cond. R } & RMSE & CV RMSE \\
\hline Bar count & -0.000530 & -0.000898 & -0.000179 & 0.003 & $<0.001$ & 0.515 & 0.117 & $0.181[0.00$ \\
\hline Note count & 0.000037 & -0.000428 & 0.000495 & 0.860 & $<0.001$ & 0.434 & 0.097 & $0.139[0.00$ \\
\hline Melodic expectations & -0.000146 & -0.000193 & -0.000094 & $<0.001$ & 0.001 & 0.701 & 0.056 & $0.111[0.00$ \\
\hline Tone transitions & -0.000075 & -0.000190 & 0.000029 & 0.187 & $<0.001$ & 0.495 & 0.102 & $0.155[0.00$ \\
\hline Pitch entropy & -0.000001 & -0.000009 & 0.000009 & 0.913 & $<0.001$ & 0.617 & 0.054 & $0.094[0.00$ \\
\hline Pitch-pair entropy & -0.000012 & -0.000021 & -0.000001 & 0.020 & $<0.001$ & 0.551 & 0.067 & $0.108[0.00$ \\
\hline Novelty & -0.000011 & -0.000026 & 0.000005 & 0.170 & $<0.001$ & 0.202 & 0.119 & $0.141[0.00$ \\
\hline
\end{tabular}

Results of setting-level models predicting measures of tune complexity from popularity ( $\mathrm{N}$ set adds, $n=12422)$. Post. means $=$ mean estimates from posterior distributions, $1-95 \% \mathrm{Cl}=1$ lower $95 \%$ credible intervals from posterior distributions, $u-95 \% \mathrm{Cl}=$ upper $95 \%$ credible intervals from posterior distributions, $p M C M C=$ ' $p M C M C$ value', marg. $R^{2}=$ marginal $R^{2}$, the proportion of variance explained by the fixed effects in the models, cond. $R^{2}=$ conditional $R^{2}$, the proportion of variance explained by both the fixed and the random effects, RMSE = normalised root mean squared error for the full model and CV RMSE = mean and SD from the distribution of normalised root mean squared error values from the CV models. All melodic complexity measures were re-scaled as the absolute deviation from the median prior to analysis, where low values indicate tunes close to median complexity. Therefore, negative coefficients here indicate that as popularity increases, tunes move towards intermediate 


\section{Figure 1}

925

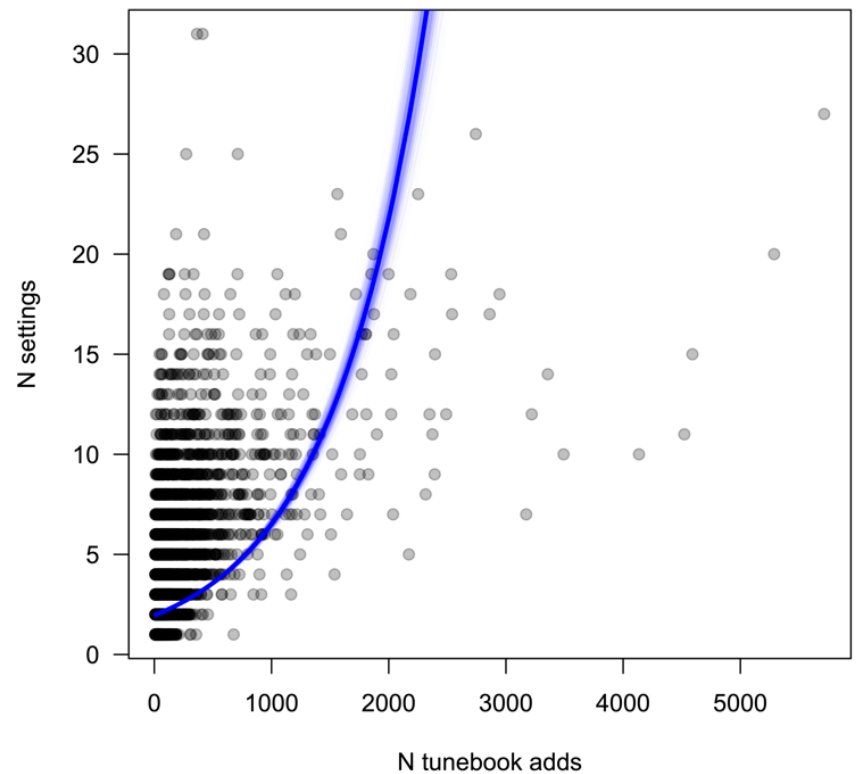

$\mathrm{N}$ tunebook adds

Scatterplot showing the relationship between tune diversity ( $N$ settings) and tune popularity ( $N$ tunebook adds), where each datapoint represents one tune $(n=9378)$. Thin blue lines represent fit lines from the entire posterior distribution, while the thick blue line is based on posterior means. Fit lines were calculated with the number of days since tunes were uploaded to the site fixed at the median value. 

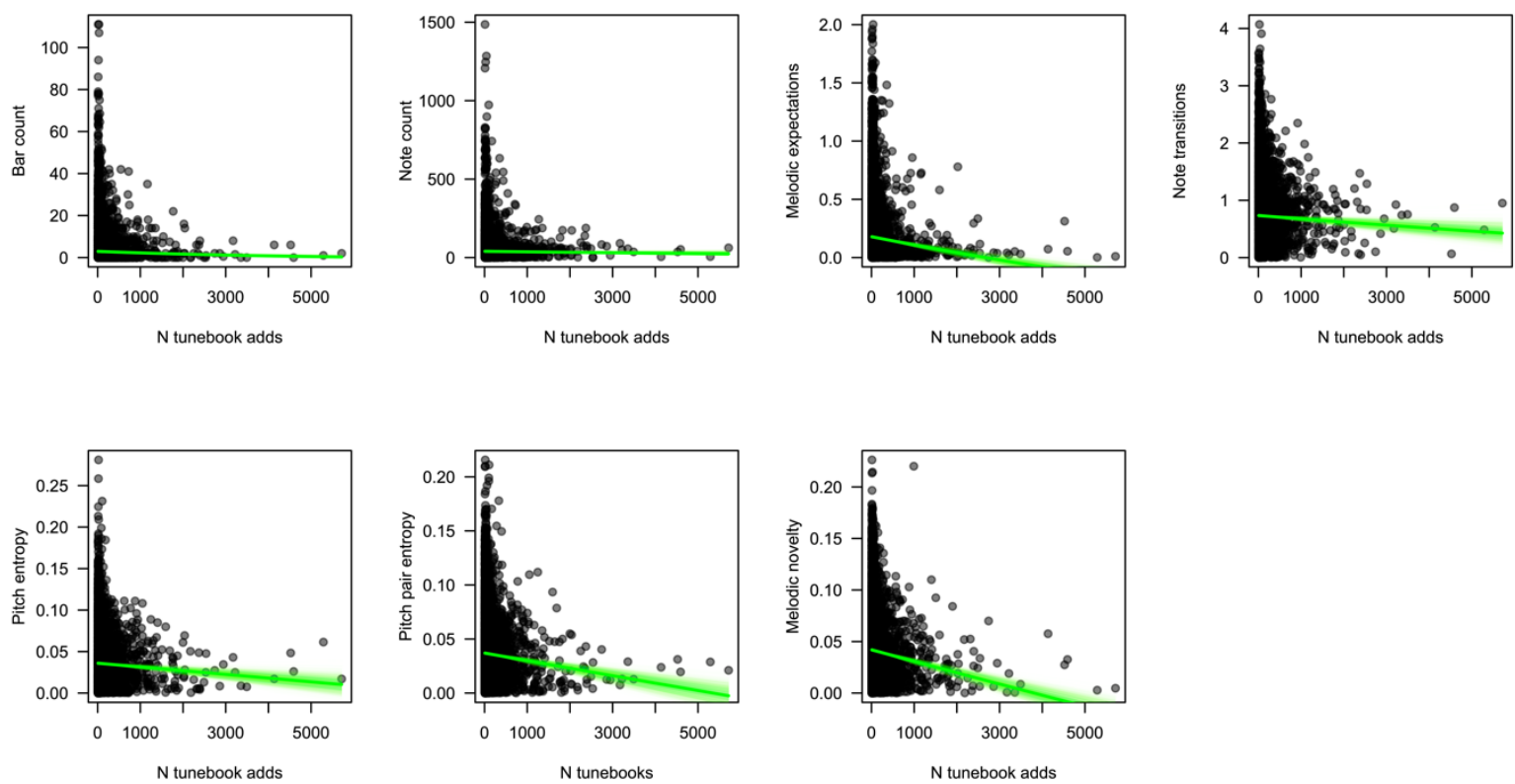

Scatterplots showing relationships between the seven measures of melodic complexity and popularity at the tune level ( $n=9378)$. Measures of complexity have been re-scaled so that they represent absolute deviation from the median values, i.e. lower values indicate tunes close to intermediate complexity while higher values tunes of higher or lower complexity. Thin green lines represent fit lines from the entire posterior distribution, while the thick green lines are based on posterior means. 
b.
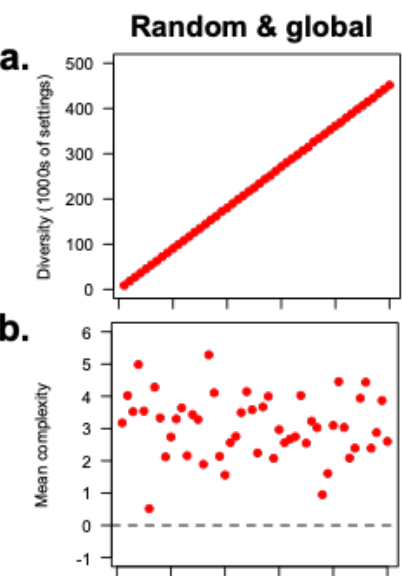

c.

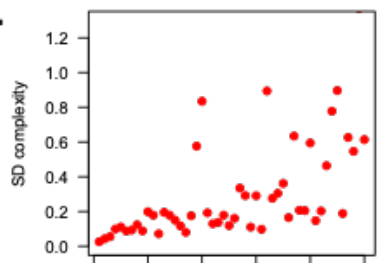

d.

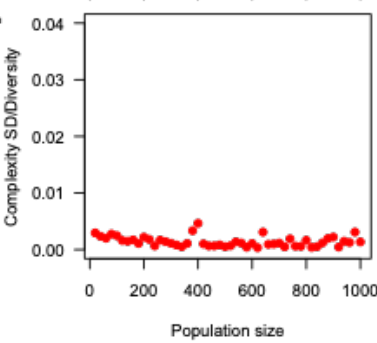

Biased \& global
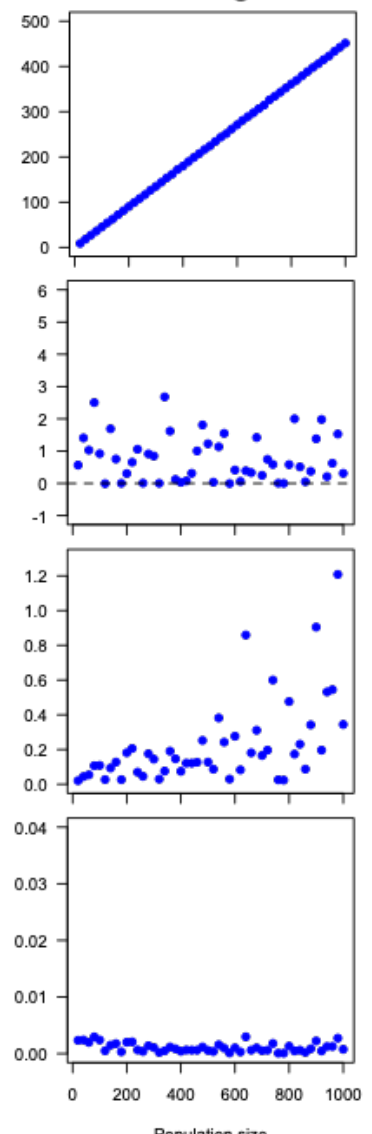

Random \& local
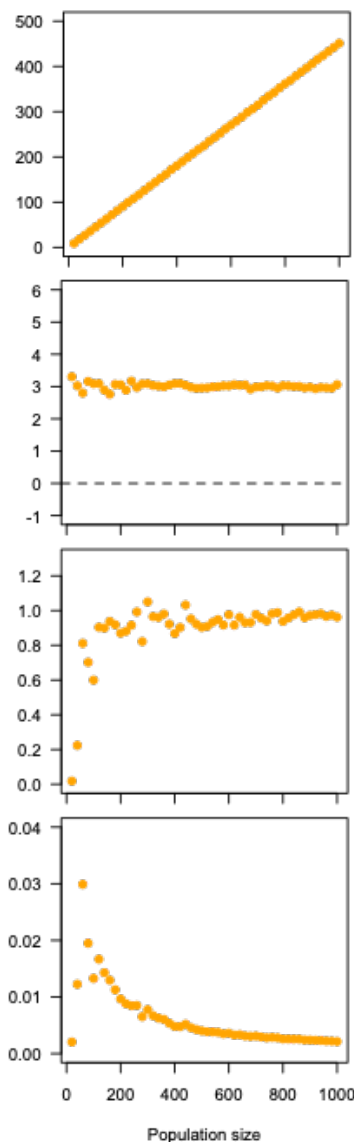

Biased \& local
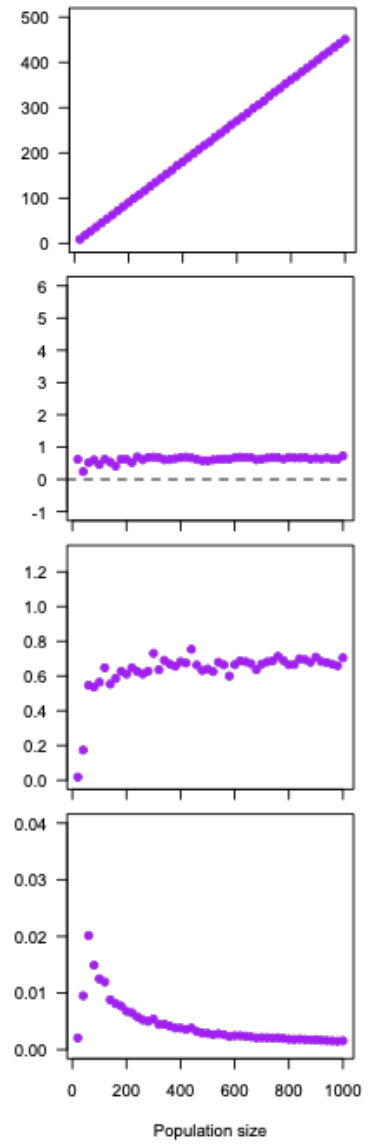

Scatterplots illustrating the relationship between population size and a. diversity (1000s of unique settings) b. mean complexity, $c$. SD complexity and c. SD complexity relative to (divided by) diversity, across the four simulation conditions: random \& global = random copying with global social influence, biased \& global = intermediate-biased copying with global social influence, random \& local = random copying with local social influence, biased \& local = intermediate-biased copying with local social influence. 

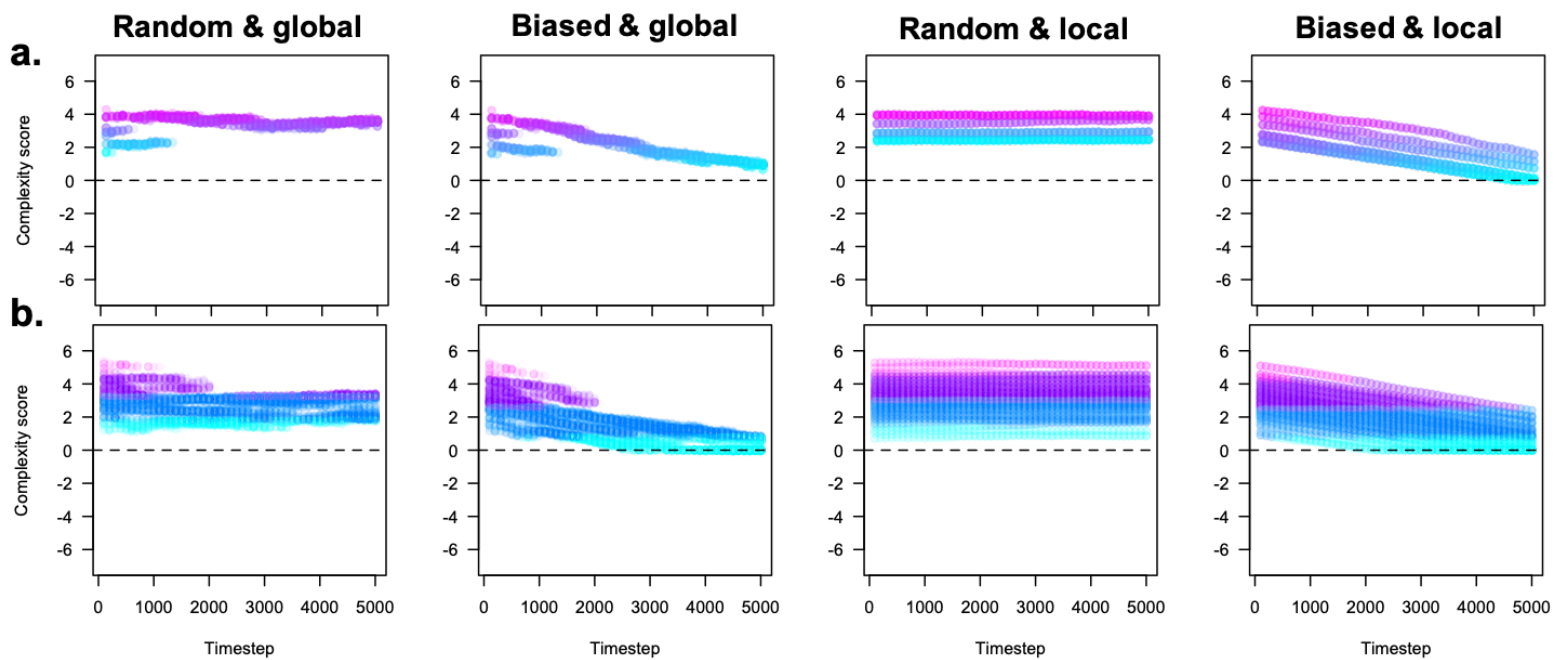

b.

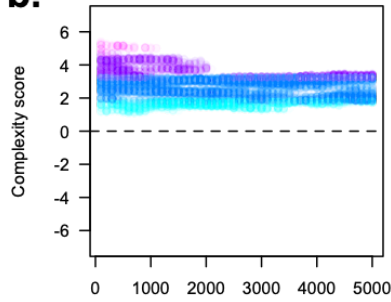

Timestep

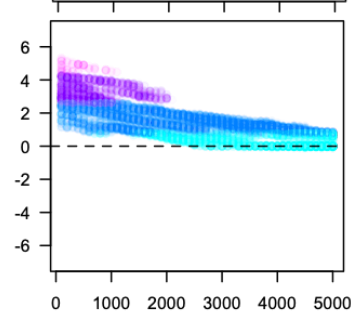

Timestep
Timestep

Plots illustrating typical trends in tune complexity in a. small $(n=100)$ and $b$. large $(n=1000)$ simulated populations across the four conditions: random \& global = random copying with global social

1030

1031

1032

1033

influence, biased \& global = intermediate-biased copying with global social influence, random \& local $=$ random copying with local social influence, biased \& local = intermediate-biased copying with local social influence. Datapoints are colour coded by complexity score for ease of visual interpretation.

1034

1035

1036

1037

1038

1039

1040

1041

1042

1043

1044

1045

1046

1047

1048

1049

1050

1051

1052

1053

1054

1055

1056 